\title{
EFFICIENT ATMOSPHERIC AND EXTRA-ATMOSPHERIC INTERCEPTION THROUGH OPTIMALLY BIASED PROPORTIONAL NAVIGATION
}

\author{
Uday S. Shukla and Pravas R. Mahapatra \\ Department of Aerospace Engineering \\ Indian Institute of Science \\ Bangalore-560012, India.
}

\begin{abstract}
Starting from a definition of the biased proportional navigation (PN) law and expression of the trajectory parameters, especially the lateral acceleration, in terms of a nondimensional bias parameter, an analytical minimization is made of the integrated (total) control effort with respect to the bias parameter. It is shown that optimum biasing may lead to significantlly more control efficient PN guidance in a wide variety of engagement situations, especially those involving higher target maneuvers. The performance of the optimally biased $\mathrm{PN}$ is compared with the standard (unbiased) PN law for the general case of a maneuvering target. The optimum bias is expressed through a simple algebraic equation readily solvable in real time even on small on-board processors. For the special (and very useful) case of the effective navigation being equal to 3 , the equation reduces to a quadratic leading to an explicit expression for the optimum bias. Specific examples are provided for interception both inside and outside the atmosphere. It is shown that control effort savings upto a factor of 3 can be achieved through optimum biasing under realistic engagement conditions.
\end{abstract}

\section{INTRODUCTION}

Pure Proportional Navigation is a commonly used pursuit strategy for guided weapons as well as for rendezvous/docking/intercept in space. In this strategy, the turning rate of the guided body is controlled to be proportional to the turn rate of the line of sight (LOS)from the guided body to the target.

Although PN guidance results in intercept under a wide variety of engagement conditions, its control-effort-efficiency is not optimum in many situations especially in the case of maneuvering targets. Scope remains for improving the efficiency. Variants have been suggested over the basic PN scheme to improve its efficiency. The biased PN $[1,2]$ is one such scheme, in which a fixed angular rate is superimposed on the measured LOS rate before computing the commanded projectile turn rate (or lateral acceleration).

Because of the introduction of an extra control parameter (i.e. the bias value), such a biased PN (BPN) may be made to achieve a given intercept with reduced total control effort. This is an important advantage for operations outside the atmosphere where lateral control forces are generated by the operation of control rockets, and the total control effort (integrated lateral force) determines the fuel requirement of the control engine(s). This fuel forms a part of the orbital payload which is at a high premium. For atmospheric flights, a reduction in control effort results in smaller pressure bottles in the case of pneumatic actuators and smaller batteries in the case of the modern all-electric actuators. The resulting space and weight saving could be very important in tactical applications.

To be able to take the best advantage of the BPN scheme, it is necessary to optimize the BPN performance with respect to the bias parameter. The performance of the BPN is maximized to obtain the optimum bias value. The development here may be considered as an extension of the earlier work by Brainin and McGhee[2]. The efficiency of BPN is explicitly compared relative to the PN which is more realistic as compared to normalization (as in Ref. 2) with respect to control effort required by a single-impulse guidance scheme which is not practical. Further, while a numerical approach was taken for the optimization in the earlier work, the optimization process in this chapter has been carried out analytically to the extent of obtaining a simple algebraic equation for the optimum bias parameter, which can be solved in real time even on small airborne computers. For the special and important case of the effective navigation constant being equal to 3 , the equation is quadratic and the optimum bias parameter is obtained in closed form. To be able to appreciate the advantages of BPN in terms of physical parameters, trajectories of BPN and PPN are plotted and examples are provided which clearly illustrate the savings in total control effort achieved by using a properly optimized BPN.

\section{DEFINITION OF BIASED PROPORTIONAL NAVIGATION}

Consider a target $T$ and a pursuer $M$ as points in a plane moving with constant speeds $V_{T}$ and $V_{M}$ respectively as shown in Fig. 1 . The line $M T$ from the pursuer to the target is the line of sight (LOS) which is inclined at an angle $\theta$ with respect to a reference line. If the pursuer velocity vector $V_{M}$ makes an angle $\phi$ with the reference line, then the standard PN law is defined as

$$
\dot{\phi}=N \dot{\theta}
$$

where $N$ is the navigation constant. , we use a modified form of (1) as follows [2] :

$$
\dot{\phi}=N\left(\theta-\theta_{B}\right)
$$

where $\theta_{B}$ is a rate bias on the LOS turn rate. Equation (2) defines the biased PN (BPN) law shown graphically in Fig. 2. The BPN law (2) reduces to the standard $\mathrm{PN}$ law (1) when $\dot{\theta}_{B}$ equals zero.

\section{SOLUTION OF BIASED PROPORTIONAL NAVIGATION}

We consider the case of pursuit against a target maneuvering with a constant lateral acceleration $A_{T}$. The governing differential equations of motion, considering the geometry only, are obtained by resolving velocity components of the target and the pursuer along and normal to the LOS.

$$
\begin{aligned}
& \dot{r}=V_{T} \cos (\theta-\beta)-V_{M} \cos (\theta-\phi) \\
& \text { and } \\
& r \dot{\theta}=-V_{T} \sin (\theta-\beta)+V_{M} \sin (\theta-\phi)
\end{aligned}
$$

where $\kappa=A_{T} / V_{T}$ represents the turn rate of the target, and $\beta=\kappa t$.

The equations (3) and (4) for the pursuer motion under PN are 


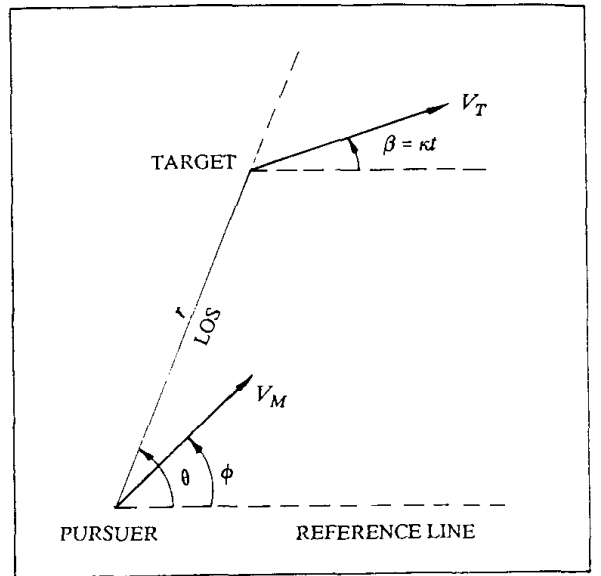

Figure 1. Geometry of Proportional Navigation

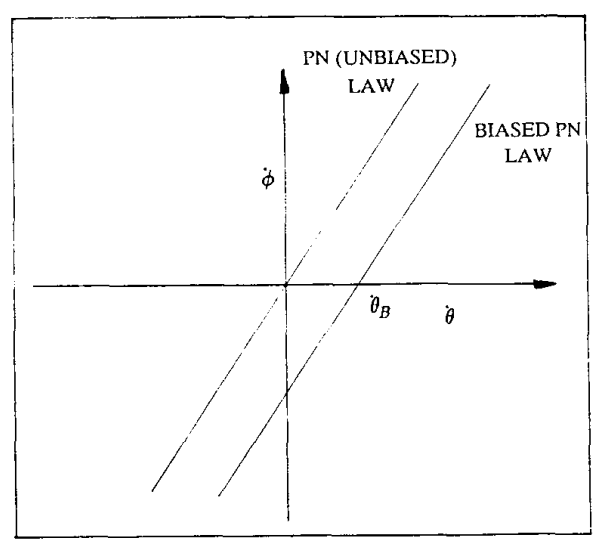

Figure 2. Biased PN scheme

not solvable in closed form. Here PN equations are linearized to make analytical treatment possible. Considering the homing trajectory to be a perturbation over a collision course, we can write (Fig. 1)

$\phi=\phi_{C}+\Delta \phi$

Assuming $\Delta \phi$ and $\theta$ to be small, (3) and (4) may be readily combined to yield an equation in $\theta$ only

$\theta\left(t_{f}-t\right)-2 \theta=\frac{V_{M}}{V_{n L}} \cos \phi_{C} \dot{\phi}+\frac{V_{T}}{V_{n i L}} \cos \beta_{i} \dot{\beta}$

where $t$ is the time from launch, $V_{n i L}$ is the initial target-pursuer relative velocity along LOS and $t_{f}=r_{i} / V_{\mathrm{nL}}$ is the final intercept time. Using the biased PN law (2), (6) reduces to

$\ddot{\theta}\left(t_{f}-t\right)-\left(2-N^{\prime}\right) \dot{\theta}-N^{\prime} \theta_{B}=\frac{V_{T}}{V_{\text {niL }}} \cos \beta_{i} \dot{\beta}$

where $N^{\prime}=\frac{N V_{M} \cos \phi_{C}}{V_{n i}}:$ Effective Navigation Constant
Equation (7) describes the behavior of the rate of change of LOS angle $(\theta)$ and can be integrated to give

$$
\begin{gathered}
\theta_{\theta}=\left[\theta_{i}-\frac{1}{N^{\prime}-2}\left[\frac{V_{T} \cos \beta_{i} \dot{\beta}}{V_{n i L}}+N^{\prime} \theta_{B}\right]\left(\frac{t_{f}-t}{t_{f}}\right)^{N^{\prime}-2}\right. \\
+\frac{1}{N^{\prime}-2}\left[\frac{V_{T} \cos \beta_{i} \dot{\beta}}{V_{n L}}+N^{\prime} \theta_{B}\right]
\end{gathered}
$$

where $\theta_{i}$ : Initial value of LOS angular rate.

Assuming $\dot{\beta}_{i}=0$ (i.e. initial target velocity direction coincides with the reference line) without loss of generality, and using $\dot{\beta}=A_{T} / V_{T}$, (by definition of $\beta$ ) eq (8) reduces to

$$
\begin{gathered}
\theta=\left[\theta_{i}-\frac{1}{N^{\prime}-2}\left(\frac{A_{T}}{V_{n i L}}+N^{\prime} \theta_{B}\right)\right]\left(\frac{t_{f}-t}{t_{f}}\right)^{N^{\prime}-2} \\
+\frac{1}{N^{\prime}-2}\left[\frac{A_{T}}{V_{n i L}}+N^{\prime} \theta_{B}\right]
\end{gathered}
$$

The expression (9) represents the LOS turn rate for pursuit against a maneuvering target under the BPN law.

\section{PURSUER LATERAL ACCELERATION}

The lateral acceleration $A_{M}$ of the pursuer under the BPN is obtained as

$$
A_{M B}=V_{M} \dot{\phi}=N V_{M}\left(\theta-\theta_{B}\right)
$$

Substituting eq (9) in eq (10) and rearranging, we get

$$
\begin{aligned}
A_{M B} & =\frac{N^{\prime}}{\left(N^{\prime}-2\right) \cos \phi_{C}}\left[a-b\left(\frac{T}{T_{i}}\right)^{N^{-2}}\right] \\
& =\frac{b N^{\prime}}{\left(N^{\prime}-2\right) \cos \phi_{C}}\left[p-\left(\frac{T}{T_{i}}\right)^{N^{-2}}\right]
\end{aligned}
$$

where

$T=$ time to $g o=t_{f}-t$

$T_{i}=$ initial value of $T=t_{f}$

$a=A_{T}+2 V_{r i} \theta_{B}$

$b=A_{T^{-}}-\left(N^{\prime}-2\right) V_{r i L} \ddot{\theta}_{i}+N^{\prime} V_{r i L} \ddot{\theta}_{B}$

$p=a / b=$ bias parameter

From (12)-(13) and the definition of $p$, the rate bias $\dot{\theta}_{B}$ is expressed as

$\theta_{B}=\frac{1-p}{\left(p N^{\prime}-2\right) V_{n L}} A_{T^{+}}+\frac{p\left(N^{\prime}-2\right)}{p N^{\prime}-2} \theta_{i}$

$$
=K \frac{A_{T}}{V_{n L}}+L \theta_{i}
$$

where

$K=$ Acceleration bias coefficient $=\frac{1-p}{\left(p N^{\prime}-2\right)}$

$L=$ Rate bias coefficient $=\frac{p\left(N^{\prime}-2\right)}{p N^{\prime}-2}$

It can be seen from eq (15) that $\theta_{B}$ is singular at $p=2 / N^{\prime}$. On 


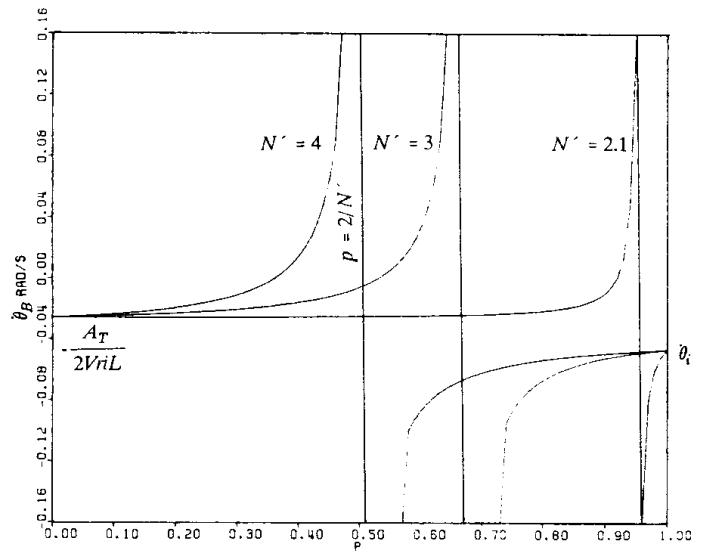

Figure 3. Behavior of the rate bias $\dot{\theta}_{\mathbf{B}}$ as a function of bias parameter $p$

either side of this singularity $\theta_{B}$ decreases and is dominated by the $A_{T}$ term for $p<2 / N^{\prime}$ and by the $\theta_{i}$ term for $p>2 / N^{\prime}$. In fact at $p=0$, $\theta_{B}=-A_{T} / 2 V_{\text {niL }}$ and at $p=1, \theta_{B}=\theta_{i}$. For the value of $p=1 /\left[1+\left(N^{\prime}-2\right) \theta_{i} V_{n L} / A_{T}\right], \theta_{B}=0$. This behavior of $\theta_{B}$ with respect to the bias parameter $p$ is depicted in Fig. 3. In addition, for $p=\infty$, $\theta_{B}=\frac{1}{N^{\prime}}\left[-A_{T} / V_{n i L}+\left(N^{\prime}-2\right) \theta_{i}\right]$.

\section{CONTROL EFFORT}

The cumulative velocity increment $\Delta V$ (which determines the total control effort) necessary for interception is defined for any pursuer trajectory as

$$
T_{1}
$$

$$
\Delta V=\int_{0}^{T_{\mathfrak{l}}}\left|A_{M}\right| d T
$$

Two cases must be considered for computing the cumulative velocity increment $\Delta V$ :

Case I : $(0 \leq p \leq 1)$

In this case exactly one change of sign of $A_{M}$ occurs in the interval $[0, T i]$. This is apparent from (11), since $p$ is a fraction between 0 and 1 , and $\left(T / T_{i}\right)^{N-2}$ decreases monotonically from 1 to 0 for $N^{\prime}>2$. The acceleration reversal occurs at

$$
\begin{aligned}
T_{r} & =T_{i}\left(\frac{a}{b}\right)^{\frac{1}{\left(N^{r}-2\right)}} \\
& =T_{i} p^{\frac{1}{\left(N^{*}-2\right)}}
\end{aligned}
$$

Then $\Delta V_{B}$ is given by

$$
\Delta V_{B}=\frac{M_{i}}{T_{i}} \frac{2 N^{\prime}}{N^{\prime}-1}\left|\frac{\left(N^{\prime}-2\right) p^{\frac{N^{\prime}-1}{N^{\prime}-2}}+\left|1+\left(N^{\prime}-2\right) p^{\frac{N^{\prime}-1}{N^{\prime}-2}}-\left(N^{\prime}-1\right) p\right|}{N^{\prime} p-2}\right|
$$

where

$$
M_{i}=\frac{1}{\cos \phi_{C}}\left|V_{n i} T_{i}^{2} \theta_{i}+\frac{A_{T}}{2} T_{i}^{2}\right|
$$

Case II : $p \leq 0$ or $p \geq 1$

Since $T / T_{i}$ has a minimum value of zero and maximum value of unity, the RHS of (11) will remain unipolar during the entire pursuit if $N^{\prime}>2$. Thus, the lateral acceleration $A_{M}$ never changes sign during the pursuit, and $\Delta V_{B}$ is given by

$\Delta V_{B}=\frac{M_{i}}{T_{i}} \frac{2 N^{\prime}\left(N^{\prime} p-p-1\right)}{\left(N^{\prime}-1\right)\left(N^{\prime} p-2\right)}$

By making use of (18) or (20), the cumulative velocity increment $\Delta V_{B}$ for BPN for any value of the bias parameter $p$ and effective navigation constant $N^{\prime}$ can be readily obtained as long as $N^{\prime}>2$, which includes most useful values of $N^{\prime}$.

\section{OPTIMUM BIASING OF PROPORTIONAL NAVIGATION}

The foregoing treatment provides a mechanism (through the introduction of a rate bias) of controlling the total control effort necessary for achieving a given mission. To make the best use of this freedom, it is necessary to optimize the rate bias to achieve a minimum control effort. Such an optimization is carried out below for the two cases considered in the last section.

\section{Case I : $0 \leq p \leq 1$}

To minimize $\Delta V_{B}$ with respect to $p$, we first examine the quantity within the inner modulus in (18) i.e.,

$$
F \equiv 1+\left(N^{\prime}-2\right) p^{\frac{N^{\prime}-1}{N^{\prime}-2}}-\left(N^{\prime}-1\right) p
$$

It can be seen that $F=1$ for $p=0$ and $F=0$ for $p=1$, and

$$
\frac{\partial F}{\partial p}=\left(N^{\prime}-1\right)\left(p^{\frac{1}{\left(N^{\prime}-2\right)}}-1\right)
$$

For $N^{\prime}>2$, the factors $\left(N^{\prime}-1\right) \operatorname{and}\left(N^{\prime}-2\right)$ are always positive. Also, since $0 \leq p \leq 1$, the quantity $p^{1 /\left(N^{-2}\right)}$ is always a fraction and hence $\left(p^{1 /(N-2)}-1\right)$ is always negative, implying that $(\partial F / \partial p)$ is always negative. We can thus remove the inner modulus from eq (18) and the problem reduces to the minimization of

$$
\Delta V_{B}=\frac{M_{i}}{T_{i}} \frac{2 N^{\prime}}{N^{\prime}-1}\left|\frac{\left(N^{\prime}-2\right) p^{\frac{N^{N}-1}{N^{\prime}-2}}+1+\left(N^{\prime}-2\right) p^{\frac{N^{\prime}-1}{N^{\prime}-2}}-\left(N^{\prime}-1\right) p}{N^{\prime} p-2}\right|
$$

Since the quantity inside the modulus in eq (23) is not necessarily unipolar, the modulus operation is likely to disturb its extremal behavior. As such, to minimize $\Delta V_{B}\left(p, N^{\prime}\right)$ from eq (23), we would first consider the quantity inside the modulus and find its extremal points in the interval $(0 \leq p \leq 1)$, and then choose the one with the least magnitude of $\Delta V_{B}\left(N^{\prime} p\right)$. Differentiating the quantity within the modulus in eq (23) and equating to zero,

$p_{o}^{N^{N}-2}-2 \frac{N^{\prime}-1}{N^{\prime}} p_{o}^{N^{-1}-2}+\frac{N^{\prime}-2}{2 N^{\prime}}=0$

Equation (24) now expresses the optimum bias parameter $p_{0}$ as a simple algebraic equation with coefficients dependent on $N^{\prime}$. In general, eq (24) will involve fractional powers of $p_{o}$, but will still be simple enough to be solved in real time even on a small airborne computer. For off-line design computation, a hand-held calculator would suffice. Once $p_{o}$ is obtained, it can be substituted for $p$ in (14) to determine the optimum rate bias $\theta_{B_{o}}$. The values of $p_{o}$ corresponding to $N^{\prime}=2.1,2.5,3,3.5,4$ and 4.5 have been obtained by a numerical solution of eq (24) and are given in Table 1 , along with the

TABLE 1. Optimum bias parameter for biased proportional navigation. $\mathrm{K}$ and $\mathrm{L}$ are the acceleration and rate bias coefficients

\begin{tabular}{|c|c|c|c|}
\hline $\mathrm{N}^{\prime}$ & $\mathrm{p}_{0}$ & $\mathrm{~K}$ & $\mathrm{~L}$ \\
\hline 2.1 & 0.787 & -0.614 & -0.227 \\
2.5 & 0.341 & -0.574 & -0.149 \\
3.0 & 0.140 & -0.544 & -0.088 \\
3.5 & 0.062 & -0.526 & -0.052 \\
4.0 & 0.029 & -0.515 & -0.031 \\
5.0 & 0.007 & -0.505 & -0.010 \\
\hline
\end{tabular}


coefficients $K$ and $L$ in eq (15). It is apparent that since $L$ is consistently much smaller than $K$, the contribution of the initial LOS rate $\dot{\theta}_{i}$ to optimum value of rate bias $\dot{\theta}_{B_{0}}$ is minor relative to that of target maneuver $A_{T}$. Further, with increasing $N^{\prime}$, the contribution due to $\theta_{i}$ becomes relatively insignificant as $L$ asymptotically approaches zero, with $K$ tending to 0.5 .

For the important special case of $N^{\prime}=3$, eq (24) reduces to the quadratic form

$6 p_{o}^{2}-8 p_{o}+1=0$

having the roots

$p_{o}=1.19$ and $p_{o}=0.14$

Since only one of the roots in (26) lies within the domain of interest $(0 \leq p \leq 1)$, the only admissible optimum corresponds to $p_{o}=0.14$.

For other integer values of $N^{\prime}$, although a closed form solution is not guaranteed, considerable simplification of eq (24) can be achieved. For example, when $N^{\prime}=4$, eq (24) reduces to

$p_{o}^{\frac{3}{2}}-1.5 p_{0}^{\frac{1}{2}}+0.25=0$

which is a simple cubic in $\left(p_{o}\right)^{1 / 2}$.

Case II : $p \leq 0$ or $p \geq 1$

The velocity increment $\Delta V_{B}$ here is given by (20). The gradjent of $\Delta V_{B}$ is given by

$$
\frac{\partial \Delta V_{B}}{\partial p}=\frac{M_{i}}{T_{i}} \frac{2 N^{\prime}\left(2-N^{\prime}\right)}{\left(N^{\prime}-1\right)\left(N^{\prime} p-2\right)^{2}}
$$

For $N^{\prime}>2$, this gradient is negative for all values of $p$, and hence $\Delta V_{B}$ is a monotonic function and has no distinct optimum. However, (28) indicates the existence of asymptotic stationary points since $\partial \Delta V_{B} / \partial p=0$ for $p \rightarrow \pm \infty$, at which $\Delta V_{B} \rightarrow 2 M_{i} / T_{i}$, from (20).

\section{Global Optimum Biasing}

To facilitate visualization of the function behavior, the dimensionless quantity $\Delta V_{B} /(M i / T i)$ is plotted in Fig. 4 for $N^{\prime}=2.1$,
3 and 4 with $p$ varying from -2 to $+3 . N^{\prime}=2$ is not plotted since eq (18) is singular when $N^{\prime}$ equals 2 . For $0 \leq p \leq 1$, the formula (18) is used, and outside this domain (20) is used. It is seen that for $p \leq 0$, the asymptotic stationary point represents a maximum and that for $p \geq 1$, it represents a minimum. that

Also an analysis of the behavior of $\Delta V_{B}$ at $p=0$ and $p=1$ shows

- $\Delta V_{B}$ at $p=0$ has a value $\left(M_{i} / T_{i}\right)\left[N^{\prime} /\left(N^{\prime}-1\right)\right]$ which is less than the asymptotic value $2 M_{i} / T_{i}$ for all $N^{\prime}>2$. Also the gradient of $\Delta V_{B}$ at $p=0$ is $\left(M_{i} / T_{i}\right)\left[N^{\prime}\left(2-N^{\prime}\right) / 2\left(N^{\prime}-1\right)\right]$ (from (28)), which is negative for all $N^{\prime}>2$.

- $\Delta V_{B}$ at $p=1$ has a value $\left(M_{i} / T_{i}\right)\left[2 N^{\prime} /\left(N^{\prime}-1\right)\right]$ which is greater than the asymptotic value $2 M_{i} / T_{i}$ for all $N^{\prime}>2$. Also, the gradient of $\Delta V_{B}$ at $p=1$ is $\left(M_{i} / T_{i}\right)\left[2 N^{\prime} /\left(N^{\prime}-1\right)\left(2-N^{\prime}\right)\right]$ (from (28)) which is negative for all $N^{\prime}>2$.

From the above argument and an examination of Fig. 4 it is clear that the global minimum of $\Delta V_{B}$ will be within the domain $0 \leq p \leq 1$, and will occur at the optimum rate bias parameter $p_{o}$ given by (24).

The singularity in $\Delta V_{B}$ in the region $0 \leq p \leq 1$ seen in Fig. 4 is predictable from eq (18) to occur at $p=2 / N^{\prime}$ and is in fact a direct result of relation (15) between the rate bias $\theta_{B}$ and bias parameter $p$ which is the variable of optimization. However, the singularity imposes no practical problems since it corresponds to infinite rate bias, as seen by letting $p=2 / N^{\prime}$ in eq (14) and also seen in Fig. 3 .

\section{RESULTS AND DISCUSSIONS}

For reasons of generality, a non-dimensional rate bias parameter $p$ has been used in the formulation. It has also resulted in improving the tractability of the problem. However, the transformation used in the non-dimensionalization has resulted in a certain blurring of the physical insight into the behavior of the biased PN system. To be able to visualize the potential benefits of the biasing in clearer focus, two specific examples are provided below.

The first example considers an air-to-air tactical situation with a

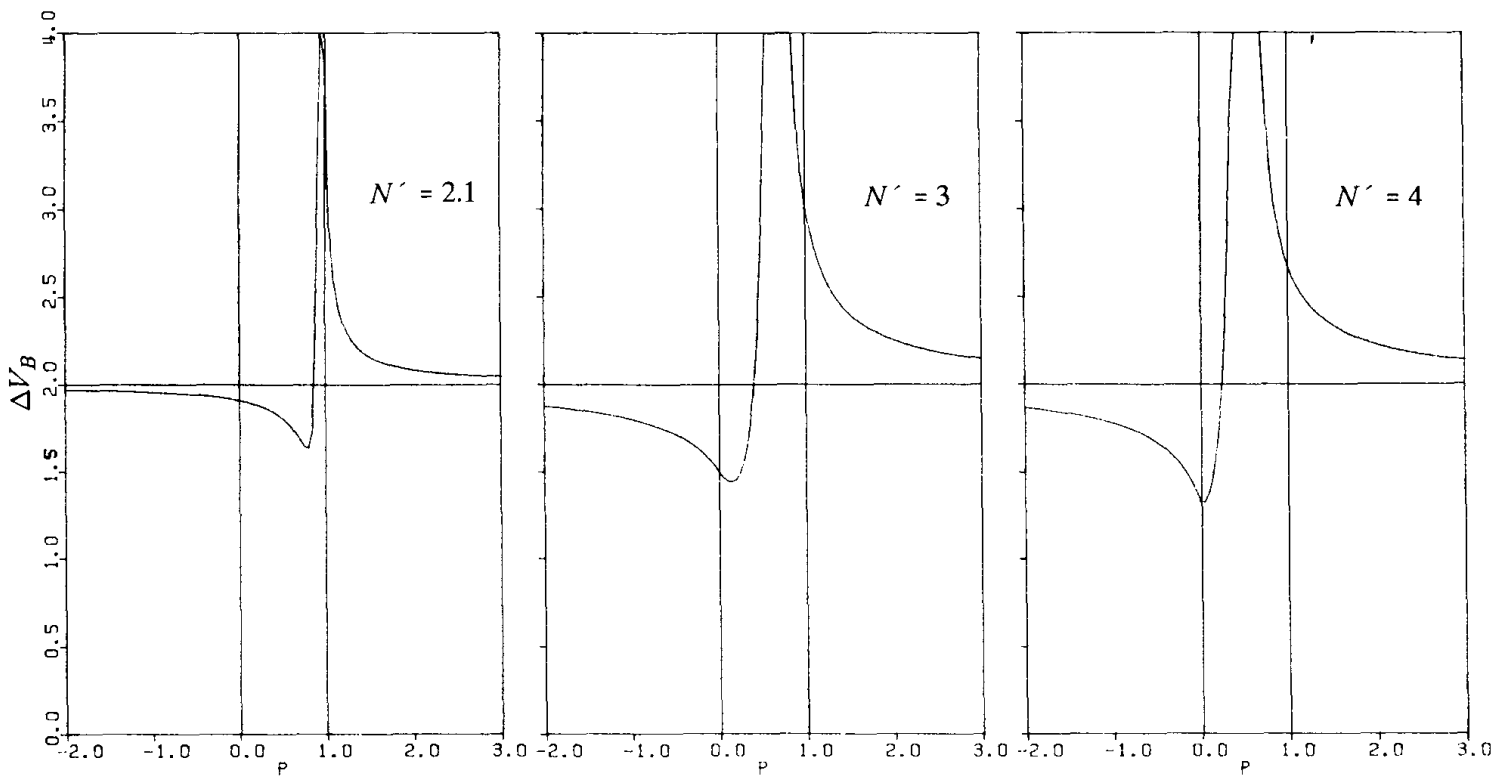

Figure 4. Normalized cumulative velocity increment $\Delta \mathrm{V}$ as a function of bias parameter $p$, illustrating the existence of global minimum within the domain $0 \leq \mathrm{p} \leq 1$. 
target speed of $300 \mathrm{~m} / \mathrm{s}$, a pursuer speed of $900 \mathrm{~m} / \mathrm{s}$, an initial pursuer-target separation of $5000 \mathrm{~m}$, and an initial LOS angle of $60^{\circ}$. Table 2 shows the optimum rate bias $B_{B_{0}}$ from (14) for a range of realistic values of the effective navigation constant $N^{\prime}$. This computation requires knowledge of the target maneuver $A_{T}$ and the initial LOS rate $\theta_{i}$ (or, equivalently, initial heading error $\Delta \phi_{i}$ ). Here, both the non-maneuvering and maneuvering targets are considered. For the non-maneuvering target case shown in Table 2 (a) two representative values of heading error $\left(\Delta \phi_{i}=15^{\circ}\right.$ and $\left.40^{\circ}\right)$ are considered. For the maneuvering target case the target lateral acceleration of $0.5 g, 1 g, 2 g, 3 g$ and $4 g$ are considered, with heading error $\Delta \phi_{i}$ taking two values, $0^{\circ}$ and $15^{\circ}$ in each case. The results are shown in Table 2 (b) through (f). The cumulative velocity increment $\Delta V_{B}$ required for intercept using BPN is computed from (18). For comparison, the cumulative velocity increment $\Delta V_{P N}$ for standard $P N$ is also obtained from (18) with $\theta_{B}=0$ and is tabulated alongside.
The optimum rate bias $\theta_{B o}$ exhibits a strong dependence on the effective navigation constant $N^{\prime}$ for a given level of target maneuver and initial heading error. The most important observation from Table 2 , however, concerns the cumulative velocity increments. It is clear from Table 2 that the optimally biased PN always requires lower total control effort than a standard PN for all maneuver levels and effective navigation constants, with or without heading error. Depending on the combination of heading error, target maneuver and $N^{\prime}$, an optimum BPN could score over a classical PN in terms of required total control effort by a factor of $1: 3$ as seen from Table 2 (f) for $N^{\prime}=5, A_{T}=4, \Delta \phi_{i}=15^{\circ}$.

While the results in Table 2 provide the behavior with respect to the total control effort, derived from the pursuer lateral acceleration, it is worthwhile viewing the optimum BPN vis-a-vis the classical PN in a more primary parameter domain such as the trajectory itself during the engagement. Both the non-maneuvering and the maneuvering

TABLE 2. Cumulative velocity increment $(\Delta V)$ requirement for biased proportional navigation for air-to-air engagement. The requirement for basic $\mathrm{PN}$ is also shown for comparison.

\begin{tabular}{|c|c|c|c|c|c|c|}
\hline \multicolumn{7}{|c|}{ Non-maneuvering target } \\
\hline \multirow{3}{*}{$N^{\prime}$} & \multicolumn{3}{|c|}{$\Delta \phi_{i}=15^{\circ}$} & \multicolumn{3}{|c|}{$\Delta \phi_{\mathrm{i}}=40^{\circ}$} \\
\hline & \multirow[t]{2}{*}{$\begin{array}{l}\dot{\theta}_{\mathrm{Bo}} \\
\mathrm{mrad} / \mathrm{s}\end{array}$} & \multicolumn{2}{|c|}{$\begin{array}{l}\Delta V \\
m / s\end{array}$} & \multirow[t]{2}{*}{$\begin{array}{c}\dot{\theta}_{\mathrm{Bo}} \\
\mathrm{mrad} / \mathrm{s}\end{array}$} & \multicolumn{2}{|c|}{$\begin{array}{l}\Delta V \\
\mathrm{~m} / \mathrm{s}\end{array}$} \\
\hline & & $\mathrm{BPN}$ & PPN & & BPN & PPN \\
\hline 2.1 & 10.51 & 378.918 & 442.654 & 27.86 & 1004.459 & 1173.416 \\
\hline 2.5 & 6.89 & 355.732 & 386.444 & 18.27 & 942.997 & 1024.410 \\
\hline 3.0 & 4.15 & 334.241 & 347.800 & 10.99 & 886.028 & 921.969 \\
\hline 3.5 & 2.42 & 318.291 & 324.613 & 6.41 & 843.745 & 860.505 \\
\hline 4.0 & 1.43 & 306.121 & 309.155 & 3.78 & 811.484 & 819.528 \\
\hline 5.0 & 0.50 & 289.106 & 289.833 & 1.31 & 766.380 & 768.308 \\
\hline
\end{tabular}

(a)

\begin{tabular}{|c|c|c|c|c|c|c|}
\hline \multicolumn{7}{|c|}{ Maneuvering target } \\
\hline \multirow{3}{*}{$N^{\prime}$} & \multicolumn{3}{|c|}{$\mathrm{A}_{\mathrm{T}}=0.5 \mathrm{~g} \quad \Delta \phi_{\mathrm{i}}=0^{\circ}$} & \multicolumn{3}{|c|}{$\mathrm{A}_{\mathrm{T}}=0.5 \mathrm{~g} \quad \Delta \phi_{\mathrm{i}}=15^{\circ}$} \\
\hline & \multirow[t]{2}{*}{$\begin{array}{c}\dot{\theta}_{\mathrm{BO}} \\
\mathrm{mrad} / \mathrm{s}\end{array}$} & \multicolumn{2}{|c|}{$\begin{array}{l}\Delta V \\
\mathrm{~m} / \mathrm{s}\end{array}$} & \multirow[t]{2}{*}{$\begin{array}{c}\dot{\theta}_{\mathrm{Bo}} \\
\mathrm{mrad} / \mathrm{s}\end{array}$} & \multicolumn{2}{|c|}{$\begin{array}{l}\Delta V \\
\mathrm{~m} / \mathrm{s}\end{array}$} \\
\hline & & BPN & PPN & & BPN & PPN \\
\hline 2.1 & -4.23 & 28.158 & 65.789 & 6.50 & 352.183 & 380.779 \\
\hline 2.5 & -3.96 & 26.435 & 57.435 & 3.13 & 330.633 & 337.196 \\
\hline 3.0 & -3.75 & 24.838 & 51.691 & 0.58 & 310.658 & 310.859 \\
\hline 3.5 & -3.63 & 23.653 & 48.245 & -1.02 & 295.833 & 296.654 \\
\hline 4.0 & -3.55 & 22.748 & 45.948 & -1.95 & 284.522 & 287.929 \\
\hline 5.0 & -3.48 & 21.484 & 43.076 & -2.81 & 268.707 & 278.012 \\
\hline
\end{tabular}

(b)

\begin{tabular}{|c|c|c|c|c|c|c|}
\hline \multicolumn{7}{|c|}{ Maneuvering target } \\
\hline \multirow{3}{*}{$\mathrm{N}^{\prime}$} & \multicolumn{3}{|c|}{$\mathrm{A}_{\mathrm{T}}=1.0 \mathrm{~g} \quad \Delta \phi_{\mathrm{i}}=0^{\circ}$} & \multicolumn{3}{|c|}{$\mathrm{A}_{\mathrm{T}}=1.0 \mathrm{~g} \quad \Delta \phi_{\mathrm{i}}=15^{\circ}$} \\
\hline & \multirow[t]{2}{*}{$\begin{array}{c}\dot{\theta}_{\mathrm{BO}} \\
\mathrm{mrad} / \mathrm{s}\end{array}$} & \multicolumn{2}{|c|}{$\begin{array}{l}\Delta V \\
m / s\end{array}$} & \multirow[t]{2}{*}{$\begin{array}{c}\dot{\theta}_{\mathrm{Bo}} \\
\mathrm{mrad} / \mathrm{s}\end{array}$} & \multicolumn{2}{|c|}{$\begin{array}{l}\Delta V \\
m / s\end{array}$} \\
\hline & & BPN & PPN & & $\mathrm{BPN}$ & PPN \\
\hline 2.1 & -8.45 & 56.316 & $131 . \overline{578}$ & 2.48 & 325.448 & 329.761 \\
\hline 2.5 & -7.92 & 52.870 & 114.869 & -0.63 & 305.533 & 305.775 \\
\hline 3.0 & -7.51 & 49.676 & 103.382 & -2.98 & 287.076 & 292.852 \\
\hline 3.5 & -7.25 & 47.306 & 96.490 & -4.47 & 273.376 & 286.630 \\
\hline 4.0 & -7.10 & 45.497 & 91.896 & -5.32 & 262.923 & 283.269 \\
\hline 5.0 & -6.97 & 42.968 & 86.152 & -6.12 & 248.309 & 280.242 \\
\hline
\end{tabular}

(c)

\begin{tabular}{|c|c|c|c|c|c|c|}
\hline \multicolumn{7}{|c|}{ Maneuvering target } \\
\hline \multirow{3}{*}{$\mathbf{N}^{\prime}$} & \multicolumn{3}{|c|}{$\mathrm{A}_{\mathrm{T}}=2.0 \mathrm{~g} \quad \Delta \phi_{\mathrm{i}}=0^{\circ}$} & \multicolumn{3}{|c|}{$\mathrm{A}_{\mathrm{T}}=2.0 \mathrm{~g} \quad \Delta \phi_{\mathrm{i}}=15^{\circ}$} \\
\hline & \multirow[t]{2}{*}{$\begin{array}{c}\dot{\theta}_{\mathrm{Bo}} \\
\mathrm{mrad} / \mathrm{s}\end{array}$} & \multicolumn{2}{|c|}{$\begin{array}{l}\Delta V \\
m / s\end{array}$} & \multirow[t]{2}{*}{ 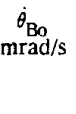 } & \multicolumn{2}{|c|}{$\begin{array}{l}\Delta V \\
m / s\end{array}$} \\
\hline & & BPN & PPN & & BPN & PPN \\
\hline 2.1 & -16.91 & 112.632 & 263.155 & -5.55 & 271.978 & 290.601 \\
\hline 2.5 & -15.83 & 105.740 & 229.739 & -8.14 & 255.335 & 290.987 \\
\hline 3.0 & -15.02 & 99.352 & 206.765 & -10.11 & 239.910 & 293.144 \\
\hline 3.5 & -14.50 & 94.611 & 192.981 & -11.35 & 228.461 & 295.757 \\
\hline 4.0 & -14.21 & 90.994 & 183.791 & -12.06 & 219.726 & 298.390 \\
\hline 5.0 & -13.93 & 85.936 & 172.304 & -12.73 & 207.513 & 303.253 \\
\hline
\end{tabular}

(d)

\begin{tabular}{|c|c|c|c|c|c|c|}
\hline \multicolumn{7}{|c|}{ Maneuvering target } \\
\hline & \multicolumn{3}{|c|}{$\mathrm{A}_{\mathrm{T}}=3.0 \mathrm{~g} \quad \Delta \phi_{\mathrm{i}}=0^{\circ}$} & \multicolumn{3}{|c|}{$\mathrm{A}_{\mathrm{T}}=3.0 \mathrm{~g} \Delta \phi_{\mathrm{i}}=15^{\circ}$} \\
\hline & \multirow[t]{2}{*}{$\mathrm{mrad} / \mathrm{s}$} & \multicolumn{2}{|c|}{$\mathrm{m} / \mathrm{s}$} & \multirow[t]{2}{*}{$\mathrm{mrad} / \mathrm{s}$} & \multicolumn{2}{|c|}{$\mathrm{m} / \mathrm{s}$} \\
\hline & & BPN & PPN & & BPN & PPN \\
\hline 2.1 & -25.36 & 168.948 & 394.733 & -13.57 & 218.507 & 313.324 \\
\hline 2.5 & -23.75 & 158.610 & 344.608 & -15.66 & 205.137 & 317.919 \\
\hline 3.0 & -22.53 & 149.028 & 310.147 & -17.24 & 192.744 & 323.350 \\
\hline 3.5 & -21.75 & 141.917 & 289.471 & -18.24 & 183.546 & 328.355 \\
\hline 4.0 & -21.31 & 136.490 & 275.687 & -18.81 & 176.528 & 332.906 \\
\hline 5.0 & -20.90 & 128.904 & 258.456 & -19.35 & 166.716 & 340.766 \\
\hline
\end{tabular}

(e)

\begin{tabular}{|c|c|c|c|c|c|c|}
\hline \multicolumn{7}{|c|}{ Maneuvering target } \\
\hline \multirow{3}{*}{$N^{\prime}$} & \multicolumn{3}{|c|}{$\mathrm{A}_{\mathrm{T}}=4.0 \mathrm{~g} \quad \Delta \phi_{\mathrm{i}}=0^{\circ}$} & \multicolumn{3}{|c|}{$\mathrm{A}_{\mathrm{T}}=4.0 \mathrm{~g} \quad \Delta \phi_{\mathrm{i}}=15^{\circ}$} \\
\hline & \multirow[t]{2}{*}{$\begin{array}{c}\dot{\theta}_{\mathrm{BO}} \\
\mathrm{mrad} / \mathrm{s}\end{array}$} & \multicolumn{2}{|c|}{$\begin{array}{l}\Delta V \\
\mathrm{~m} / \mathrm{s}\end{array}$} & \multirow[t]{2}{*}{$\dot{\mathrm{\theta}}_{\mathrm{Bo}} \mathrm{\textrm {mrad } / \mathrm { s }}$} & \multicolumn{2}{|c|}{$\begin{array}{l}\Delta V \\
\mathrm{~m} / \mathrm{s}\end{array}$} \\
\hline & & BPN & PPN & & BPN & PPN \\
\hline 2.1 & -33.82 & 225.264 & 526.311 & -21.60 & 165.037 & 370.642 \\
\hline 2.5 & -31.66 & 211.481 & 459.478 & -23.17 & 154.939 & 369.265 \\
\hline 3.0 & -30.03 & 198.705 & 413.530 & -24.37 & 145.579 & 371.573 \\
\hline 3.5 & -29.01 & 189.222 & 385.961 & -25.12 & 138.631 & 375.320 \\
\hline 4.0 & -28.42 & 181.987 & 367.582 & -25.55 & 133.331 & 379.420 \\
\hline 5.0 & -27.86 & 171.872 & 344.608 & -25.96 & 125.920 & 387.365 \\
\hline
\end{tabular}

(f) 
target cases are considered. In Fig. 5 the trajectory of a pursuer following biased PN guidance law against a non-maneuvering target is plotted for the initial conditions corresponding to results in Table 2 (a). Two most commonly used $N^{\prime}$ values of 3 and 4 are used. Next, a laterally maneuvering target is considered and pursuer trajectories are plotted for the moderate and high target maneuvers corresponding to the initial conditions in Table 2 (d) and (f) respectively. Fig. 6 corresponds to $N^{\prime}=3$ while a value of $N^{\prime}=4$ is used in Fig. 7. It is seen from these plots that the BPN trajectory for the pursuer is always flatter (i.e. less curved) as compared to the standard PN. As anticipated from the results of Table 2, the cases showing a larger saving in control effort result in a higher level of flattening of the optimal BPN trajectory relative to the standard PN. In the case of non-maneuvering targets, this flattening takes the trajectory close to the ideal case of collision course and in the case of maneuvering targets the flattening takes the trajectory towards the line joining the start of the engagement and the intercept point.

The second example corresponds to an engagement scenario in extra-atmospheric space. The initial target-pursuer separation is 185 $\mathrm{km}$ and the relative initial closing speed is $9000 \mathrm{~m} / \mathrm{s}$. These values are the same as those used for illustration in [1]. Here also, an initial LOS angle of $60^{\circ}$ is assumed, as also an "initial miss distance" of 15 $\mathrm{km}$, corresponding to an initial LOS rate of $3.944 \mathrm{mrad} / \mathrm{s}$. In Table 3 , in addition to the cumulative velocity increments $\Delta V_{B_{0}}$ and $\Delta V_{P N}$, the quantity of propellant required for effecting these velocity increments are also presented. The latter quantity is computed assuming, as in [1], an initial interceptor weight of $270 \mathrm{~kg}$ and a liquid propellant with a specific impulse of $300 \mathrm{~s}$. Either the cumulative velocity increment or the propellant requirement can be taken as a measure of the required control effort.

TABLE 3. Cumulative velocity increment and propellant required for biased proportional navigation for extra-atmospheric engagement. The requirement for basic PN is also shown for comparison.

\begin{tabular}{|c|c|c|c|c|c|}
\hline \multicolumn{6}{|c|}{$\begin{array}{c}\text { Non-maneuvering target } \\
\dot{\dot{\theta}_{\mathrm{i}}}=3.94 \mathrm{mrad} / \mathrm{s}\end{array}$} \\
\hline \multirow[t]{2}{*}{$\mathrm{N}^{\prime}$} & \multirow[t]{2}{*}{$\begin{array}{c}\theta_{\mathrm{Bo}} \\
\mathrm{mrad} / \mathrm{s}\end{array}$} & \multicolumn{2}{|c|}{$\begin{array}{l}\Delta V \\
\mathrm{~m} / \mathrm{s}\end{array}$} & \multicolumn{2}{|c|}{$\begin{array}{c}\text { PROPELLANT } \\
\mathrm{Kg}\end{array}$} \\
\hline & & BPN & PPN & $\mathrm{BPN}$ & PPN \\
\hline 2.1 & .89 & 1192.383 & 1392.949 & 91.611 & 103.692 \\
\hline 2.5 & .59 & 1119.421 & 1216.067 & 87.007 & 93.080 \\
\hline 3.0 & .35 & 1051.795 & 1094.460 & 82.637 & 85.406 \\
\hline 3.5 & .21 & 1001.601 & 1021.496 & 79.328 & 80.647 \\
\hline 4.0 & .12 & 963.305 & 972.853 & 76.766 & 77.408 \\
\hline 5.0 & .04 & 909.761 & 912.050 & 73.126 & 73.283 \\
\hline
\end{tabular}

(a)

\begin{tabular}{|c|c|c|c|c|c|}
\hline & & $\begin{aligned} & \text { Maneu } \\
&= 3.94 \mathrm{mI} \\
&\end{aligned}$ & $\begin{array}{l}\text { ring targe } \\
1 / \mathrm{s} \quad \mathrm{A}_{\mathrm{T}}=\end{array}$ & & \\
\hline $\mathbf{N}^{\prime}$ & $\mathrm{\theta}_{\mathrm{Bo}}$ & & & $\overline{\mathrm{PROPI}}$ & LANT \\
\hline & & $\mathrm{BPN}$ & PPN & $B P N$ & PPN \\
\hline 2.1 & .56 & 1109.999 & 1202.128 & 86.404 & 92.217 \\
\hline 2.5 & .27 & 1042.078 & 1063.782 & 82.001 & 83.419 \\
\hline 3.0 & .06 & 979.124 & 979.945 & 77.828 & 77.883 \\
\hline 3.5 & -.08 & 932.398 & 934.640 & 74.673 & 74.825 \\
\hline 4.0 & -.16 & 896.748 & 906.771 & 72.231 & 72.921 \\
\hline 5.0 & -.23 & 846.904 & 875.028 & 68.768 & 70.729 \\
\hline
\end{tabular}

(b)

\begin{tabular}{|c|c|c|c|c|c|}
\hline & & $\begin{aligned} & \text { Maneu } \\
&= 3.94 \mathrm{~m} \\
&\end{aligned}$ & $\begin{array}{l}\text { ring targe } \\
1 / \mathrm{s} \quad \mathrm{A}_{T}=\end{array}$ & & \\
\hline $\mathrm{N}^{\prime}$ & $\begin{array}{c}\hat{\theta}_{\mathrm{Bo}} \\
\mathrm{mrad} / \mathrm{s}\end{array}$ & & & PROPI & LANT \\
\hline & & BPN & PPN & $\mathrm{BPN}$ & PPN \\
\hline 2.1 & .23 & 1027.614 & 1043.073 & 81.050 & 82.066 \\
\hline 2.5 & .04 & 964.734 & 965.160 & 76.862 & 76.890 \\
\hline 3.0 & -.24 & 906.453 & 922.973 & 72.899 & 74.030 \\
\hline 3.5 & -.37 & 863.195 & 902.514 & 69.906 & 72.628 \\
\hline 4.0 & -.44 & 830.191 & 891.353 & 67.593 & 71.859 \\
\hline 5.0 & -.51 & 784.046 & 881.078 & 64.316 & 71.149 \\
\hline
\end{tabular}

(c)

\begin{tabular}{|c|c|c|c|c|c|}
\hline \multicolumn{6}{|c|}{$\begin{array}{l}\text { Maneuvering target } \\
\dot{\theta}_{\mathrm{i}}=3.94 \mathrm{mrad} / \mathrm{s} \quad \mathrm{A}_{\mathrm{T}}=2.0 \mathrm{~g}\end{array}$} \\
\hline \hline $\mathrm{N}^{\prime}$ & $\begin{array}{c}\dot{\theta}_{\mathrm{Bo}} \\
\mathrm{mrad} / \mathrm{s}\end{array}$ & \multicolumn{2}{|c|}{$\Delta \mathrm{V}$} \\
$\mathrm{m} / \mathrm{s}$ & \multicolumn{2}{c|}{$\begin{array}{c}\text { PROPELLANT } \\
\mathrm{Kg}\end{array}$} \\
\cline { 3 - 7 } & & $\mathrm{BPN}$ & $\mathrm{PPN}$ & $\mathrm{BPN}$ & $\mathrm{PPN}$ \\
\hline 2.1 & -.44 & 862.845 & 914.950 & 69.882 & 73.482 \\
2.5 & -.67 & 810.048 & 914.500 & 66.169 & 73.451 \\
3.0 & -.83 & 761.111 & 920.206 & 62.667 & 73.841 \\
3.5 & -.94 & 724.789 & 927.756 & 60.031 & 74.357 \\
4.0 & -1.00 & 697.077 & 935.562 & 57.997 & 74.888 \\
5.0 & -1.06 & 658.331 & 950.201 & 55.121 & 75.881 \\
\hline
\end{tabular}

(d)

\begin{tabular}{|c|c|c|c|c|c|}
\hline & & $\begin{aligned} & \text { Manel } \\
&= 3.94 \mathrm{~m} \\
&\end{aligned}$ & $\begin{array}{l}\text { ring targe } \\
\mathrm{d} / \mathrm{s} \quad \mathrm{A}_{\mathrm{T}}=\end{array}$ & & \\
\hline$N^{\prime}$ & $\begin{array}{c}\theta_{\mathrm{Bo}} \\
\mathrm{mrad} / \mathrm{s}\end{array}$ & & & $\overline{\mathrm{PROP}}$ & LANT \\
\hline & & $\mathrm{BPN}$ & PPN & BPN & PPN \\
\hline 2.1 & -1.11 & 698.076 & 977.692 & 58.071 & 77.732 \\
\hline 2.5 & -1.29 & 655.361 & 992.509 & 54.899 & 78.723 \\
\hline 3.0 & -1.43 & 615.769 & 1009.680 & 51.918 & 79.865 \\
\hline 3.5 & -1.51 & 586.383 & 1025.361 & 49.679 & 80.902 \\
\hline 4.0 & -1.56 & 563.963 & 1039.564 & 47.956 & 81.836 \\
\hline 5.0 & -1.61 & 532.616 & 1064.026 & 45.525 & 83.435 \\
\hline
\end{tabular}

(e)

\begin{tabular}{|c|c|c|c|c|c|}
\hline \multicolumn{6}{|c|}{$\begin{array}{c}\text { Maneuvering target } \\
\dot{\theta}_{i}=3.94 \mathrm{mrad} / \mathrm{s} \quad \mathrm{A}_{\mathrm{T}}=4.0 \mathrm{~g}\end{array}$} \\
\hline \multirow[t]{2}{*}{$\mathrm{N}^{\prime}$} & \multirow[t]{2}{*}{$\begin{array}{c}\theta_{\mathrm{Bo}} \\
\mathrm{mrad} / \mathrm{s}\end{array}$} & \multicolumn{2}{|c|}{$\begin{array}{l}\Delta \mathrm{V} \\
\mathrm{m} / \mathrm{s}\end{array}$} & \multicolumn{2}{|c|}{$\begin{array}{c}\text { PROPELLANT } \\
\mathrm{Kg}\end{array}$} \\
\hline & & $\mathrm{BPN}$ & PPN & BPN & PPN \\
\hline 2.1 & -1.78 & 533.307 & 1148.531 & 45.579 & 88.857 \\
\hline 2.5 & -1.92 & 500.674 & 1146.493 & 43.021 & 88.729 \\
\hline 3.0 & -2.02 & 470.428 & 1155.072 & 40.624 & 89.271 \\
\hline 3.5 & -2.09 & 447.978 & 1167.494 & 38.830 & 90.053 \\
\hline 4.0 & -2.13 & 430.849 & 1180.715 & 37.451 & 90.882 \\
\hline 5.0 & -2.16 & 406.901 & 1205.947 & 35.510 & 92.454 \\
\hline
\end{tabular}

(f) 

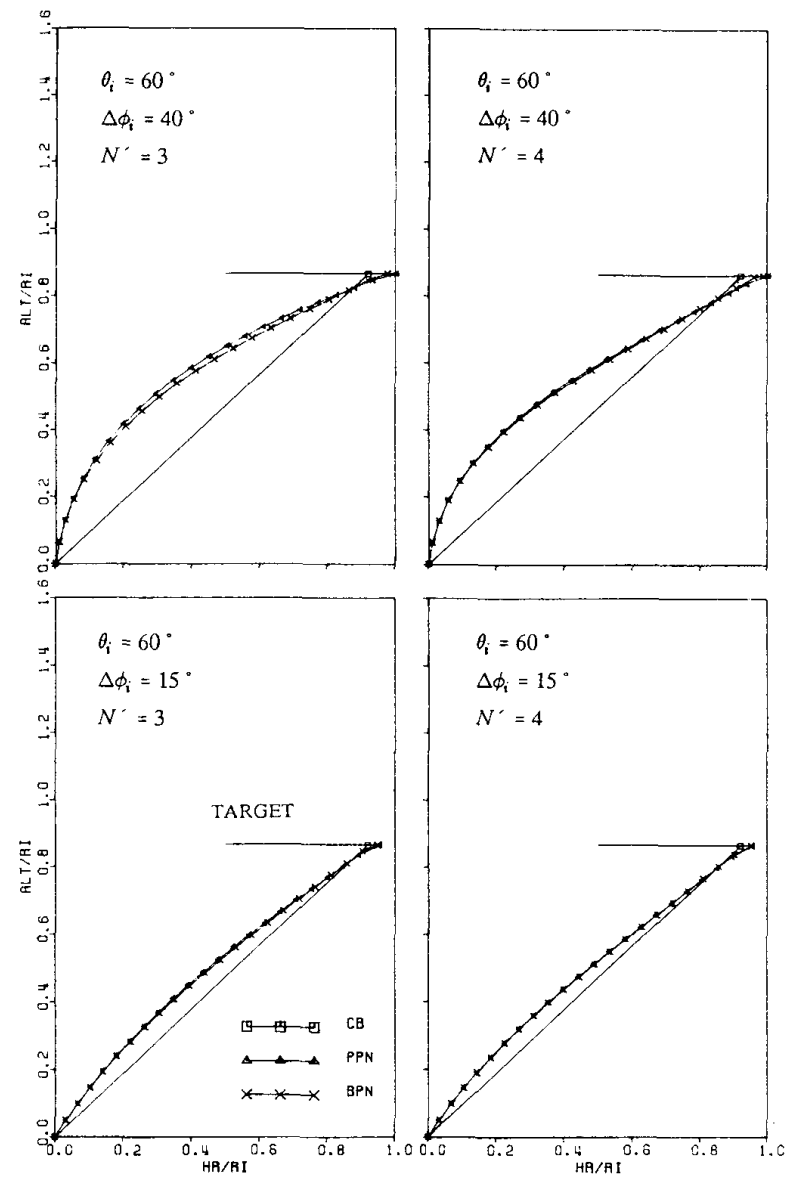

Figure 5. Pursuer trajectory for a biased PN law against a nonmaneuvering target. The trajectory under PPN and the Collision course are also shown for comparison.

In section (a) of Table 3 a non-maneuvering space target is considered while in sections (b) and (c), target maneuver $A_{T}$ value of $0.5 \mathrm{~g}$ and $1 \mathrm{~g}$ are assumed. It is apparent that in space pursuit scenarios, control effort can be saved by employing optimum BPN even for relatively low target maneuvers. Thus, for a nonmaneuvering target, a $13 \%$ propellant saving over the standard PN is possible if $N^{\prime}=2.1$ is used while it is $3 \%$ for commonly used value of $N^{\prime}=3$. A saving of about $3 \%$ is possible for $A_{T}=0.5 \mathrm{~g}$ and it increases to about $10 \%$ over the standard PN, for $A_{T}=1.0 \mathrm{~g}$ and $N^{\prime}=5$. Target maneuver $A_{T}$ is progressively increased in section (d) and (e) of Table 3 and it is seen that the saving is as high as $27 \%$ for $A_{T}=2 \mathrm{~g}$ and $45 \%$ for $A_{T}=3 g$ using $N^{\prime}=5$. In Table 3(f), a high target maneuver of $4 g$ is deliberately included, keeping in view the possible space-based pursuit-evasion applications of the near future. For such target maneuvers, the propellant saving is by a factor of $1: 2$ to $1: 2.6$ for $N^{\prime}$ varying from 2.1 to 5.0 .

\section{CONCLUSIONS}

Biased Proportional Navigation (BPN) has been studied from the point of view of control effort requirement. It has been shown that

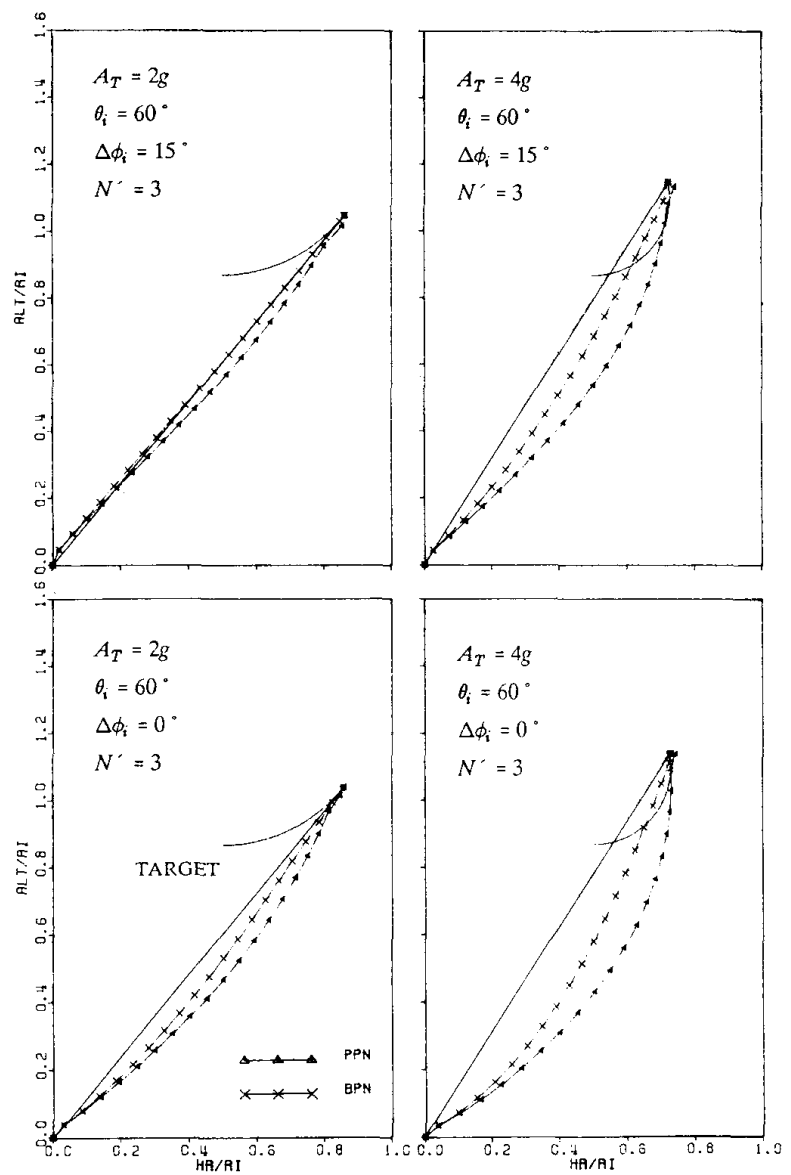

Figure 6. Pursuer trajectory for a biased PN law against a laterally maneuvering target. The trajectory under PPN is also shown for comparison. $N^{\prime}=3$.

with optimal choice of the rate bias, it is possible to effect large savings in control effort required for intercepting maneuvering targets.

An analytical optimization of the BPN problem has been carried out in terms of a non-dimensional rate bias parameter, resulting in a simple algebraic equation for the optimum value of the parameter from a minimum-control-effort point of view. The equation can be easily solved in real time even in the simple on-board computers of small projectiles. For the special but very useful case of $N^{\prime}=3$, the solution for the optimum rate bias parameter is explicit.

Two examples have been provided to concretely illustrate the gains possible by using an optimal BPN over the standard PN both in terms of total control effort and the trajectory behavior. The examples concern both atmospheric and extra-atmospheric pursuits. It has been shown that for highly maneuvering targets, the optimal BPN may require a total control effort as low as one third of the effort necessary for PN without bias. Such savings can be extremely valuable especially in extra-atmospheric engagements where maneuvers are carried out at the direct expense of propellant which forms part of the precious payload. 


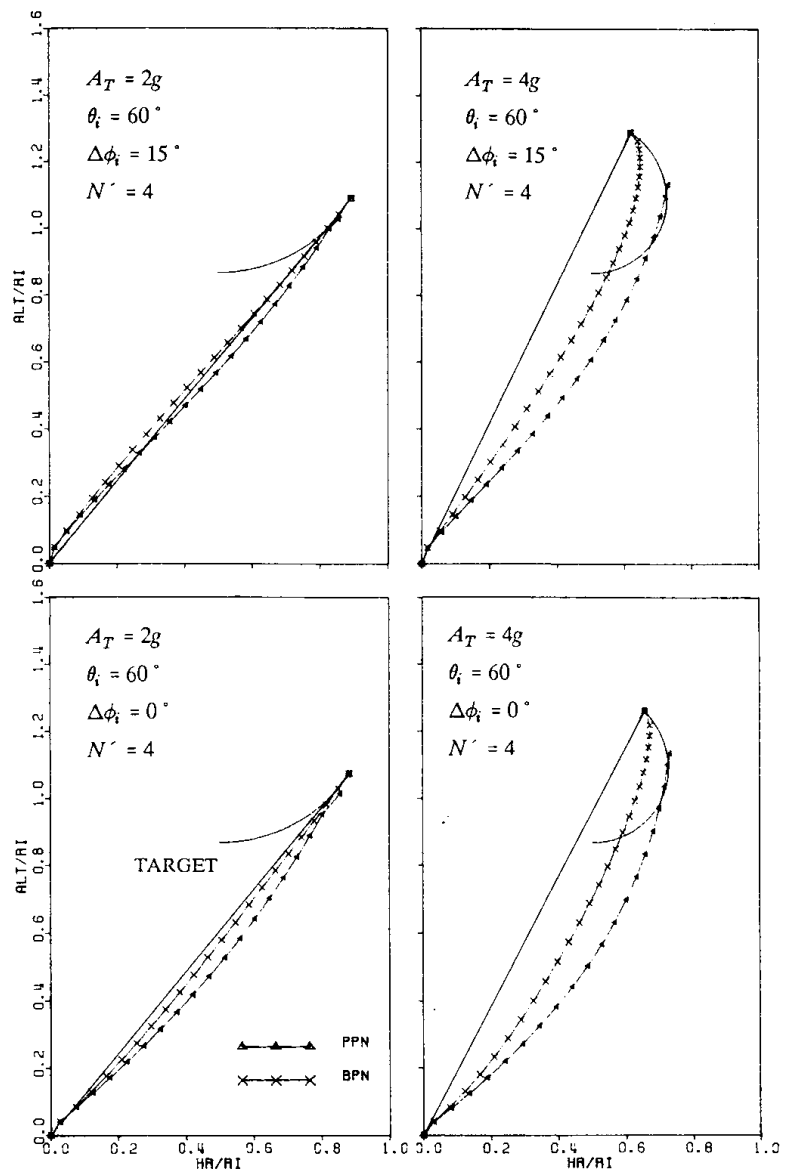

Figure 7. Pursuer trajectory for a biased PN law against a laterally maneuvering target. The trajectory under PPN is also shown for comparison. $\mathrm{N}^{\prime}=4$.

\section{REFERENCES}

[1] Murtaugh, S.A. and Criel, H.E. (1966): "Fundamentals of Proportional Navigation," IEEE Spectrum, Vol. 3, No. 12, Dec. 1966, pp. 75-85.

[2] Brainin, S.M. and McGhee, R.B. (1968): "Optimal Biased Proportional Navigation," IEEE Transactions on Automatic Control, Vol. AC-13, No. 4, Aug. 1968, pp. 440-442. 